\title{
Intrinsic Limits of Subthreshold Slope in Biased Bilayer
}

\section{Graphene Transistor}

\author{
Kausik Majumdar, Kota V. R. M. Murali ${ }^{\dagger}$, Navakanta Bhat and Yu-Ming Lin ${ }^{\dagger \dagger}$ \\ Department of Electrical Communication Engineering and \\ Center of Excellence in Nanoelectronics, \\ Indian Institute of Science, Bangalore-560012, India. \\ ${ }^{\dagger}$ IBM Semiconductor Research and Development Center, Bangalore - 560045, India. \\ ${ }^{\dagger}$ IBM T. J. Watson Research Center, Yorktown Heights, NY 10598, USA.
}

\begin{abstract}
In this work, we investigate the intrinsic limits of subthreshold slope in a dual gated bilayer graphene transistor using a coupled self-consistent Poisson-bandstructure solver. We benchmark the solver by matching the bias dependent bandgap results obtained from the solver against published experimental data. We show that the intrinsic bias dependence of the electronic structure and the self-consistent electrostatics limit the subthreshold slope obtained in such a transistor well above the Boltzmann limit of $60 \mathrm{mV} /$ decade at room temperature, but much below the results experimentally shown till date, indicating room for technological improvement of bilayer graphene.
\end{abstract}

\footnotetext{
${ }^{*}$ Corresponding author: Kausik Majumdar, Email: kausik@ece.iisc.ernet.in
} 
The excellent transport properties of single and multi-layer graphene hold promise to build ultra-fast transistors with excellent on state characteristics [1]-[4]. However, the lack of significant bandgap in such systems has been one of the major roadblocks to achieve low off state current and hence high on/off current ratio [2]-[4. Recently, it has been found, both theoretically and experimentally, that a bandgap can be opened up in a bilayer graphene (BLG) using an external bias [4]-[11]. A recent experiment shows that a bandgap as large as $\sim 0.3 \mathrm{eV}$ can be created in a BLG depending on the external bias [6]. This relatively large bandgap is promising to obtain low off state current, hence an improved on to off current ratio and subthreshold slope [1].

The aim of this paper is to discuss the intrinsic limits of the subthreshold slope in a BLG metal-oxide-semiconductor field effect transistor (MOSFET). We assume a long channel transistor with an ideal gate dielectric interface. To achieve this, we develop a self-consistent Poisson-bandstructure solver for a dual gated BLG device. We have simulated the magnitude of the bias dependent bandgap tuning in a BLG system which matches closely with the published experimental data [6] validating the solver. We also show that the gate bias dependent electronic structure and the self-consistent electrostatics play a central role in determining the intrinsic limits of the subthreshold slope in a BLG transistor, which remains well above the room temperature Boltzmann limit of $60 \mathrm{mV} /$ decade.

A schematic of the BLG device configuration is shown in Fig. 1(a) where a BLG is sandwiched between a top and a bottom gate stack. Each gate stack consists of a gate dielectric with an equivalent oxide thickness (EOT) of $1 \mathrm{~nm}$ and a gate contact metal. We assume perfect dielectric-BLG interface and zero flatband voltage. The voltage at the top gate $\left(V_{t}\right)$ and the bottom gate $\left(V_{b}\right)$ can be varied independently. The self-consistent electronic structure of this device configuration is determined by the bandstructure of the BLG using Tight Binding Method ([1, 9, 10]) coupled with the 1-D Poisson equation. Taking the center of the two graphene layers at $z=0$, the charge density is given by $\rho(z)=q n(z)$ where $q$ is the electronic charge and $n(z)$ is obtained as a subtraction between the hole $\left(n_{h}(z)\right)$ and electron $\left(n_{e}(z)\right)$ carrier density:

$$
n(z)=2\left[\sum_{i, \bar{k}}\left(1-f\left(E_{i}(\bar{k})\right)\right)\left|\psi_{i}^{\bar{k}}(z)\right|^{2}-\sum_{j, \bar{k}} f\left(E_{j}(\bar{k})\right)\left|\psi_{j}^{\bar{k}}(z)\right|^{2}\right]
$$


where $f\left(E_{i, j}(k)\right)$ is the Fermi-Dirac probability of the state $(\{i, j\}, \bar{k})$ at temperature $T$. $i$ and $j$ are the valence and conduction band indices respectively. The energy eigenvalues $E_{i, j}(\bar{k})$ are obtained from the tight binding bandstructure taking only $p_{z}$ orbital into account, with an intra-layer overlap integral $(S)$ of 0.129 , the intra-layer hopping $t$ as $-3.033 \mathrm{eV}$ and inter-layer hopping $t_{\perp}$ as $-0.365 \mathrm{eV}$ [1]. To obtain the wavefunction $\psi_{i}^{\bar{k}}(z)$, we assume normalized Gaussian orbital as the basis function. The wavefunctions are set to zero at the BLG-dielectric interfaces.

We validate the above self-consistent method by comparing the bias dependent bandgaps of BLG experimentally obtained in [6]. In this case, $V_{t}$ is set to $-V_{b}$ which breaks the inversion symmetry of the BLG opening a bandgap [4, 5]. The results are shown in Fig. 1(b) where the bandgap $\left(E_{g}\right)$ is plotted as a function of the average displacement vector $D$, defined in the same way as in [6]. Here the relative permittivity $\left(\epsilon_{r}\right)$ of BLG is varied as a parameter to find that $\epsilon_{r}=1.8$ provides the best fit to the experimental data and the same value is used in the Poisson equation to generate the results discussed in the rest of the paper. Fig. 1(c) shows the carrier density profiles along $z$ at $D=3 \mathrm{~V} / \mathrm{nm}$. This clearly shows a strong charge polarization in the bilayer, though the system as a whole remains almost intrinsic for $V_{t}=-V_{b}$.

The recent development of significant tunability of bandgap in a bilayer graphene ([5]-[10]) brings the possibility of significant reduction of off current in a BLG transistor. In the following, we show that the bias dependent electronic structure and the corresponding self-consistent electrostatics play a major role in determining the intrinsic limits of the subthreshold slopes and the on/off current ratio in an ideal long channel BLG MOSFET.

Before discussing the self-consistent results, we first show that even in absence of the screening effect, the intrinsic bias dependence of the electronic structure in BLG can be a major cause for subthreshold slope degradation. This arises from the dependence of the rate of change of electron and hole barrier height $\left(B_{e, h}\right)$ with gate bias. This rate, ideally, should be as high as possible, with the maximum possible value being 1, which corresponds to a subthreshold slope of $60 \mathrm{mV} /$ decade. This is obtained by finding the roots $\lambda$ of the secular equation of the bilayer Hamiltonian matrix (with $S=0$ ):

$$
\begin{array}{r}
{\left[\left(V_{t}-\lambda\right)^{2}-t^{2}\left|s_{k}\right|^{2}\right]\left[\left(V_{b}-\lambda\right)^{2}-t^{2}\left|s_{k}\right|^{2}\right]} \\
+t_{\perp}^{2}\left(V_{t}-\lambda\right)\left(V_{b}-\lambda\right)=0
\end{array}
$$


where $s_{k}=1+e^{i \bar{k} \cdot \overline{a_{1}}}+e^{i \bar{k} \cdot \overline{a_{2}}}, \overline{a_{1}}$ and $\overline{a_{2}}$ are lattice vectors in graphene. In Fig. 11(d), we plot the rate of change of $B_{e}$ for different directions in the $\left(V_{t}, V_{b}\right)$ space in polar coordinates with $\theta=\tan ^{-1}\left(V_{t} / V_{b}\right)$. This clearly shows that the rate is maximum $(=1)$ along $V_{t}=V_{b}+V_{0}$, which is essentially the constant bandgap locus. As we deviate from this direction, the rate degrades from 1 with minimum along $V_{t}=-V_{b}$, leading to the poor subthreshold slope along anti-symmetric bias case. As observed from Fig. 1(d), this is a fairly generic result for a large variety of points in the subthreshold region.

We now consider a chemically undoped long channel BLG MOSFET with metal source and drain having low drain bias. The drain current is assumed to be completely dominated by the thermionic carriers, neglecting tunneling. Under these approximations, the electron barrier height $B_{e}$ is plotted in Fig. 2(a) as a function of $V_{t}$ and $V_{b}$. In the same plot, we also show a number of possible paths $p$ from transistor off state (labeled $\mathrm{X}_{p}$ points) to on state (labeled $\mathrm{Y}_{p}$ points).

Due to the ambipolar nature of the device, it is important to select the appropriate off state for the device. The total integrated carrier density $\left(N=N_{e}+N_{h}\right)$ is shown in Fig. 2(b) in the $\left(V_{t}, V_{b}\right)$ space to help choose the appropriate set of off state points. In Fig. 2(a), $\mathrm{X}_{1,4}$ corresponds to the point of maximum bandgap in that range. $\mathrm{X}_{2}$ corresponds to the maximum electron barrier height though it reduces the hole barrier height, hence increasing $N . \mathrm{X}_{3}$ is an intermediate point with symmetric electron and hole barrier heights. We now compare the characteristics among these off to on paths shown in Fig. 2(a), namely (1): $V_{b}$ is fixed at $1 \mathrm{~V}$ and $V_{t}$ is varied from $-1 \mathrm{~V}$ to $1 \mathrm{~V},(2)$ : $V_{b}$ is fixed at $0.5 \mathrm{~V}$ and $V_{t}$ is varied from $-1 \mathrm{~V}$ to $1 \mathrm{~V}$, (3): $V_{b}=V_{t}+1 \mathrm{~V}$, and $V_{t}$ is varied from $-1 \mathrm{~V}$ to $0 \mathrm{~V}$, which is an almost constant bandgap operation (with $E_{g} \approx 0.17 \mathrm{eV}$ ), (4): $V_{b}=-V_{t}$ and $V_{t}$ is varied from $-1 \mathrm{~V}$ to $0 \mathrm{~V}$, which is the maximum bandgap tunability path. It should be noted that (1) and (2) correspond to single gate operation with back gate at fixed bias. On the other hand, (3) and (4) correspond to double gate operation, although unconventional, with top and bottom gates at different biases. The conventional double gate operation with $V_{t}=V_{b}$ is of little significance due to zero bandgap. Fig. 2(c) shows $N$ as a function of $V_{t}$ in these four cases. It is clearly observed that the best on/off ratio obtained is $\sim 100$. Also, path (1) and (4) will have lower off current than (2) and (3), which is also expected from the Fig. 2(b). Path (4) is found to have low on/off ratio 
due to the poor tunability of barrier heights (though bandgap tunability is maximum) in the anti-symmetric case.

To get insights into the above, we find the drain current in a long channel transistor as $I_{d}=q N \tau$, where $\tau$ is the transport factor. In a MOSFET configuration, $\tau$ is a weak function of gate voltage. Hence, the subthreshold slope is $S S=\ln (10) \frac{\partial V_{t}}{\partial \ln \left(I_{d}\right)} \approx \ln (10) \frac{\partial V_{t}}{\partial \ln (N)}$. In Fig. 2 (d), the computed subthreshold slopes are plotted for the paths (1) to (4) with varying $V_{t}$. Note that, unlike conventional MOSFET, the computed subthreshold slope is not independent of gate bias in the BLG transistor.

Analytically, we can convert the summation used in Eq. 1 into an integral to obtain $N$ :

$$
N=2\left[\int_{-\infty}^{E_{v}} D_{v}(E)(1-f(E)) d E+\int_{E_{c}}^{\infty} D_{c}(E) f(E) d E\right]
$$

where only one band is assumed to contribute in the conduction band (CB) and the valence band $(\mathrm{VB}) . E_{v, c}$ are the $\mathrm{VB}_{\max }$ and $\mathrm{CB}_{\min }$ obtained from the self-consistent electrostatics. $D_{v, c}$ are the 2-D DOS of $\mathrm{VB}$ and $\mathrm{CB}$ respectively, and we replace them by an average DOS $D$ using the fact that close to band edge, $D_{v, c}$ change much slowly as compared to Fermi function 9]. Utilizing CB-VB symmetry, we find,

$$
N=2 k_{B} T D \ln \left[\left(1+e^{-B_{e}^{\prime}}\right)\left(1+e^{-B_{h}^{\prime}}\right)\right]
$$

where $B_{e}^{\prime}=\frac{B_{e}}{k_{B} T}=\frac{E_{c}-\mu}{k_{B} T}$ and $B_{h}=\frac{B_{h}}{k_{B} T}=\frac{\mu-E_{v}}{k_{B} T}$. As $D$ is weakly dependent on $V_{t}$ [9], we obtain

$$
S S=-\ln (10) \frac{k_{B} T \kappa \ln (\kappa)}{\left[e^{-B_{e}^{\prime} \frac{\partial B_{e}}{\partial V_{t}}}+e^{\left.-B_{h}^{\prime} \frac{\partial B_{h}}{\partial V_{t}}+e^{-E_{g}^{\prime}} \frac{\partial E_{g}}{\partial V_{t}}\right]}\right.}
$$

where $\kappa=\left(1+e^{-B_{e}^{\prime}}\right)\left(1+e^{-B_{h}^{\prime}}\right)$ and $E_{g}^{\prime}=\frac{E_{g}}{k_{B} T}=\frac{B_{e}+B_{h}}{k_{B} T}$. In a conventional Si MOSFET, in the subthreshold region, $B_{e}^{\prime}, B_{h}^{\prime}>>0, E_{g}$ is constant and no gate field screening occurs due to small carrier density $\left(\left|\frac{\partial B_{e, h}}{q \partial V_{t}}\right|=1\right)$. Under these conditions, it is straight forward to show that $S S$ reduces to $\ln (10)\left(\frac{k_{B} T}{q}\right)(=60 \mathrm{mV} /$ decade at $T=300 \mathrm{~K})$. Unfortunately, for bilayer graphene, these three conditions are never satisfied simultaneously which degrades the subthreshold slope. To open up a bandgap in BLG, unlike conventional semiconductor, we need to apply significantly large fields at the two gates. This in turn causes a large number of carriers to be present in the device, even in subthreshold (though it is possible that the overall device is close to neutral, like in the case of $V_{t}=-V_{b}$, Fig. 1(c)). These carriers cause a strong screening of the gate field 
and hence, a reduction in the derivatives in the denominator of Eq. 5. Thus, even if we create a significant bandgap using vertical field, the carriers thus generated degrade the subthreshold slope.

In the anti-symmetric bias case (path (4)), using $B_{e}=B_{h}=\frac{E_{g}}{2}$, we find from Eq. 5 that the $S S$ limit reduces to $60 /\left|\frac{\partial B_{e}}{q \partial V_{t}}\right|$. As $\left|\frac{\partial B_{e}}{q \partial V_{t}}\right|$ is intrinsically small in this direction (see Fig. 11(d)), $S S$ degrades significantly. On the other hand, along the constant bandgap paths (e.g. path $3)$, the $S S$ limit again reduces to $60 /\left|\frac{\partial B_{e}}{q \partial V_{t}}\right|$. However, in this case, the strong screening due to large number of carriers reduces the denominator, limiting $S S$ much above Boltzmann limit. Finally, we have numerically performed a global search in the $\left(V_{t}, V_{b}\right)$ space with $\left|V_{t, b}\right| \leq 1 \mathrm{~V}$, which shows that the best $S S$ for all possible constant bandgap paths is around $200 \mathrm{mV} /$ decade, whereas along all possible constant $V_{b}$ paths is around $275 \mathrm{mV} /$ decade.

Thus, we find that along the constant bandgap loci, the fundamental limit of $S S$ arising from the bias dependent electronic structure is $60 \mathrm{mV} /$ decade (see Fig. 1(d)), but the screening due to the presence of large number of carriers arising from the unavoidable gate field degrades the subthreshold slope significantly. However, as the operating condition moves away from this direction, both the electronic structure as well as the screening effect play major roles to degrade $S S$. Nonetheless, the shown subthreshold slope limits are significantly less than the most recent experimental data reported [11] where the extracted value of the minimum subthreshold slope is $\sim 550 \mathrm{mV} /$ decade. This clearly shows that there is room for significant technological improvement in bilayer graphene, including efforts in improving transport derived bandgap, effective oxide thickness and choosing the optimized path from on state to off state.

In conclusion, in this work, we have developed a coupled self-consistent Poisson-bandstructure solver for dual gated bilayer graphene. The bias dependent bandgap results obtained from the solver have been benchmarked against published experimental data. Finally, it has been shown that the bias dependent electronic structure and the self-consistent electrostatics limit the subthreshold slope obtained in such a transistor well above the Boltzmann limit of $60 \mathrm{mV} /$ decade, but again much below the results experimentally shown till date.

Two of the authors (K.M. and N.B.) would like to sincerely acknowledge the support from the Ministry of Communication and Information Technology (MCIT), Govt. of India, and the 
Department of Science and Technology (DST), Govt. of India. 


\section{References}

[1] R. Saito, G. Dresselhaus, and M. S. Dresselhaus, "Physical Properties of Carbon Nanotubes," Imperial College Press, London, 1998.

[2] M. C. Lemme, T. J. Echtermeyer, M. Baus, and H. Kurz, IEEE Elec. Dev. Lett., 28, 282 (2007).

[3] Y. Sui and J. Appenzeller, Nano Lett., 9, 2973 (2009).

[4] J. B. Oostinga, H. B. Heersche, X. Liu, A. F. Morpurgo and L. M. K. Vandersypen, Nat. Mat., 7, 151 (2008).

[5] T. Ohta, A. Bostwick, T. Seyller, K. Horn and E. Rotenberg, Science 313, 951 (2006).

[6] Y. Zhang, T. Tang, C. Girit, Z. Hao, M. C. Martin, A. Zettl, M. F. Crommie, Y. R. Shen and F. Wang, Nature, 459, 820 (2009).

[7] E. McCann, Phys. Rev. B, 74, 161403(R) (2006).

[8] E. V. Castro, K. S. Novoselov, S.V. Morozov, N. M. R. Peres, J. M. B. Santos, J. Nilsson, F. Guinea, A. K. Geim, and A. H. Castro Neto, Phys. Rev. Lett., 99, 216802 (2007).

[9] E. V. Castro, K. S. Novoselov, S.V. Morozov, N. M. R. Peres, J. M. B. Santos, J. Nilsson, F. Guinea, A. K. Geim, and A. H. Castro Neto, arXiv:0807.3348v1 [cond-mat.mes-hall] (2008).

[10] J. Nilsson, A. H. Castro Neto, F. Guinea, and N. M. R. Peres, Phys. Rev. B, 78, 045405 (2008).

[11] F. Xia, D. B. Farmer, Y. M. Lin and P. Avouris, Nano Lett., 10, 715 (2010). 

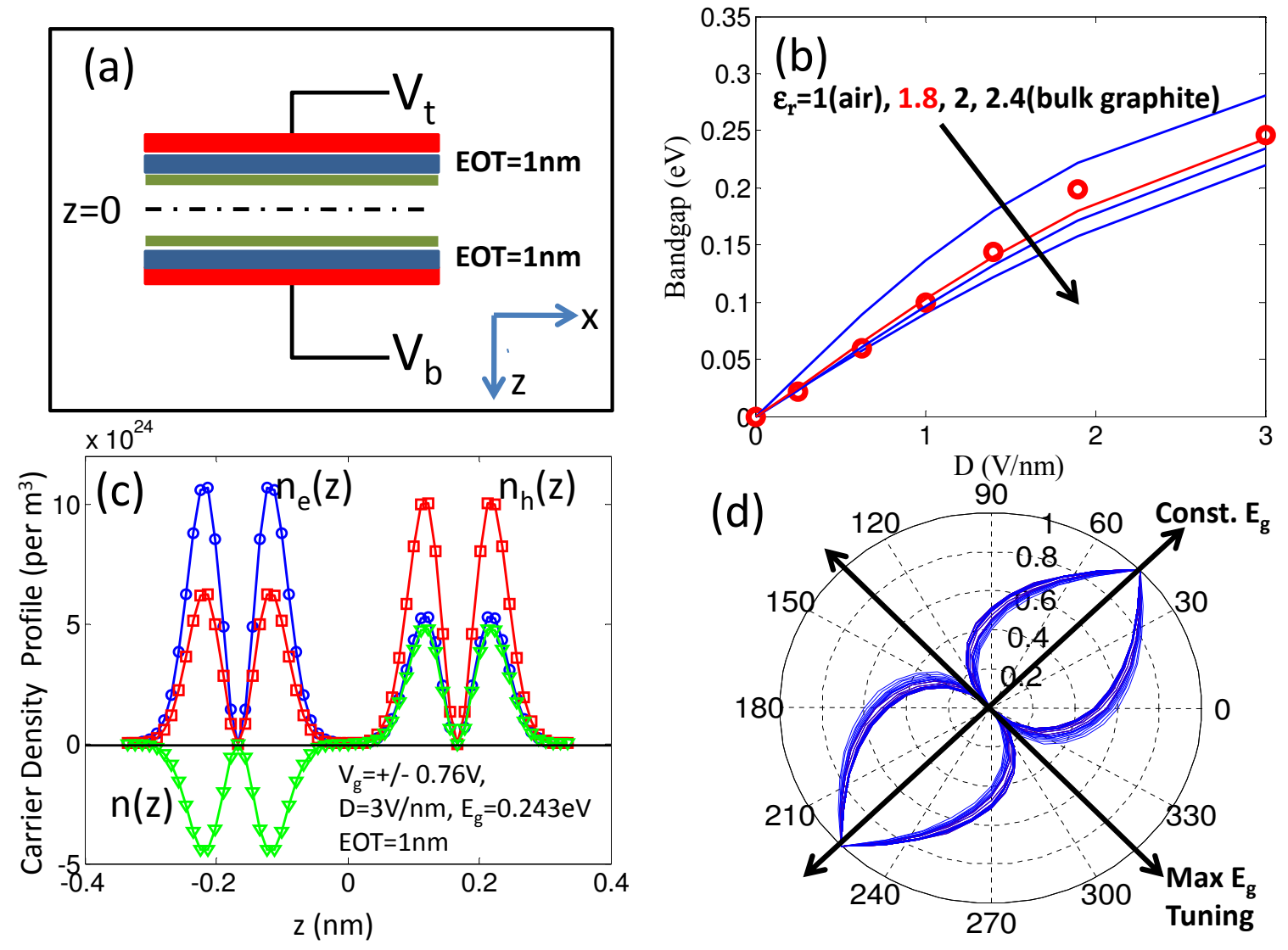

Figure 1: (a): Schematic of a bilayer graphene (BLG) sandwiched between two gate stacks. (b): Verification of numerically obtained bandgap results with experimental data in [6], shown as red dots. (c): Carrier density profiles from top layer to bottom layer. (d): Polar plot of the rate of change of $B_{e}$ (normalized by $q$ ) along different directions using Eq. 2 (with angle $\left.\theta=\tan ^{-1}\left(V_{t} / V_{b}\right)\right)$ in the $\left(V_{t}, V_{b}\right)$ space for two sets of subthreshold points: i) $V_{t}=-1 \mathrm{~V}$, $V_{b} \in[0 \mathrm{~V}, 1 \mathrm{~V}]$ and ii) $V_{t}=-0.5 \mathrm{~V}, V_{b} \in[0 \mathrm{~V}, 0.5 \mathrm{~V}]$. All the curves show similar directional dependence. 

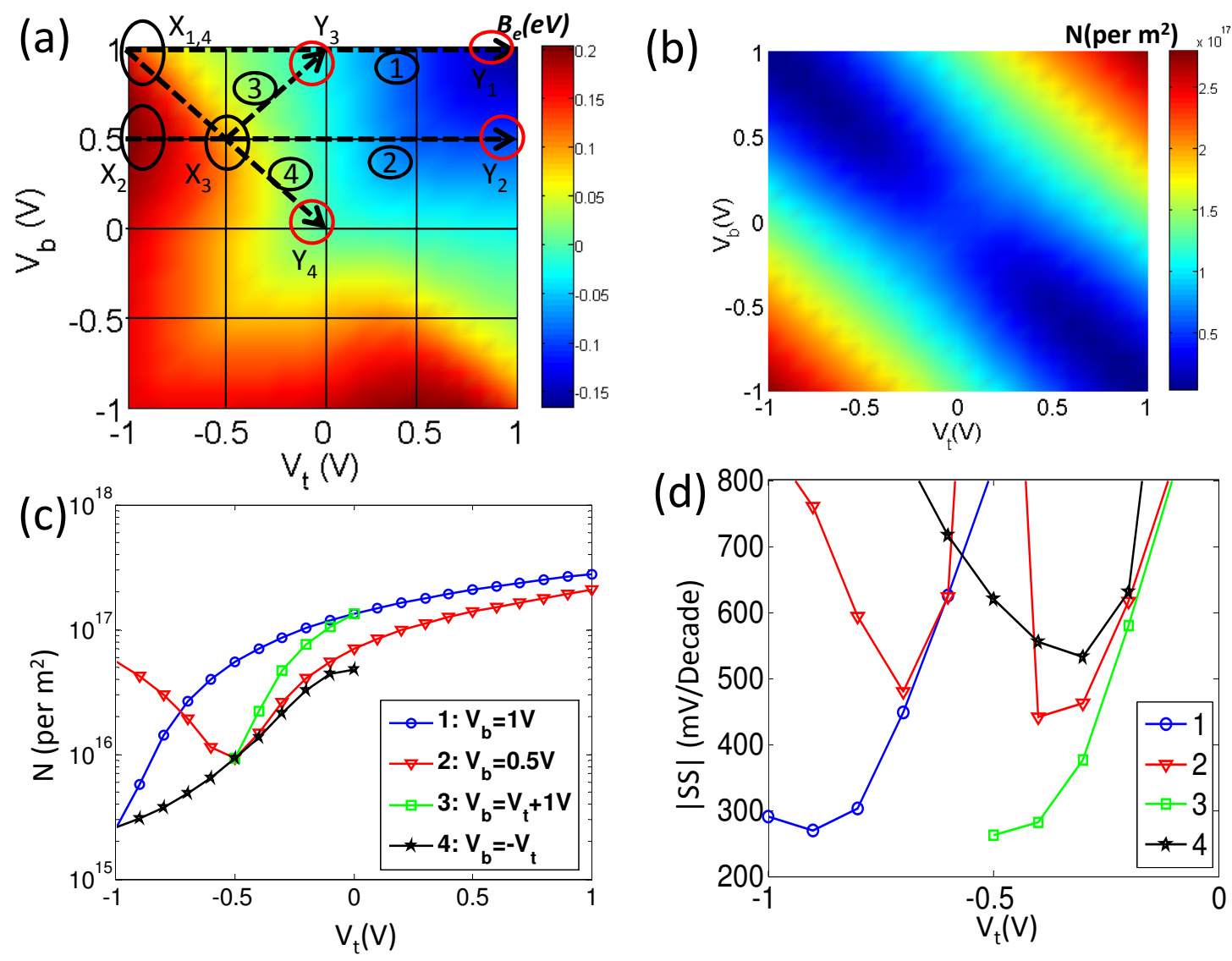

Figure 2: (a): Electron Barrier height $B_{e}$ as a function of $V_{t}$ and $V_{b}$. The four different off to on state arrow paths are numbered as (1) to (4). (b): Integrated carrier density $(N)$ as a function of $V_{t}$ and $V_{b}$. (c): $\mathrm{N}$ along the different off to on paths (d): The absolute values of the computed subthreshold slopes for the paths (1)-(4) without any non-ideality. 